\title{
Particle Beam Therapy for Cancer of the Skull Base, Nasal Cavity, and Paranasal Sinus
}

\author{
Nobuyoshi Fukumitsu \\ Department of Radiation Oncology, Ibaraki Prefectural Central Hospital, 6528, Koibuchi, Kasama 309-1793, Japan \\ Correspondence should be addressed to Nobuyoshi Fukumitsu, fukumitsun@yahoo.co.jp
}

Received 25 February 2012; Accepted 9 April 2012

Academic Editors: C. Y. Chien, S. Kanzaki, M. Tahara, and D. Thurnher

Copyright ( $) 2012$ Nobuyoshi Fukumitsu. This is an open access article distributed under the Creative Commons Attribution License, which permits unrestricted use, distribution, and reproduction in any medium, provided the original work is properly cited.

\begin{abstract}
Particle beam therapy has been rapidly developed in these several decades. Proton and carbon ion beams are most frequently used in particle beam therapy. Proton and carbon ion beam radiotherapy have physical and biological advantage to the conventional photon radiotherapy. Cancers of the skull base, nasal cavity, and paranasal sinus are rare; however these diseases can receive the benefits of particle beam radiotherapy. This paper describes the clinical review of the cancer of the skull base, nasal cavity, and paranasal sinus treated with proton and carbon ion beams, adding some information of feature and future direction of proton and carbon ion beam radiotherapy.
\end{abstract}

\section{Introduction}

Particle beam therapy was first proposed in the 1940s [1] and was investigated in the USA, Sweden, and the Soviet Union in the 1950-1960s. In the 1970s, particle beam therapy was rapidly developed in parallel with the development of X-ray computed tomography. Globally, more than 48,000 patients have been treated with particle beams. Most of these treatments were delivered using proton and carbon ion radiotherapy (RT). Currently, there are 36 particle beam therapy facilities in operation (proton: 33, carbon ion: 5, both particles: 2), according to the Particle Therapy CoOperative Group homepage. Ten facilities are located in the USA, followed by eight in Japan, three in Germany, three in Russia, two in France, and 10 in other countries (Table 1).

This paper describes the status and prospects of particle beam therapy for the treatment of cancer of the skull base, nasal cavity, and paranasal sinus, including physical, biological, technical, and financial aspects.

\section{Physical, Biological, Technical, and Financial Aspects of Particle Beam Therapy}

The physical, biological, technical, and financial aspects of particle beam therapy differ largely from those of conventional photon RT.
2.1. Physical Aspects. Both proton and carbon ion beams have features that are extremely different from those of photons. Accelerated proton and carbon ion beams show an increase in energy deposition with penetration depth up to a sharp maximum followed by rapid decrease at the end of the penetration range; this phenomenon is known as the Bragg peak (Figure 1). The particle range is determined by the energy of the incoming particles, and the Bragg peak can be spread out. These features permit a more precise and conformal dose localization to the target, compared with photon beams.

2.2. Biological Aspects. Proton and carbon ion beams lose energy when they penetrate a material. With an increasing atomic mass and electric charge of the ions, the ionization density or linear energy transfer (LET) increases. Proton beams have a higher LET than photons; however, their radiobiological properties do not differ substantially from those of photons. For clinical applications, the absorbed dose is multiplied by a factor of 1.1 to express the biological effective dose in cobalt gray equivalents (GyE). Carbon ion beams have a biological advantage over photon and proton beams. Their biological efficiency increases at the end of the beam's range, whereas it is low along the entrance channel. The local values for relative biological effectiveness (RBE) 
TABLE 1: Institution of particle beam therapy.

\begin{tabular}{|c|c|c|c|}
\hline Institution & Country & Particle & Start of treatment \\
\hline ITEP, Moscow & Russia & $\mathrm{P}$ & 1969 \\
\hline St. Petersburg & Russia & $\mathrm{P}$ & 1975 \\
\hline PSI, Villigen & Switzerland & $\mathrm{P}$ & 1996 \\
\hline Dubna & Russia & $\mathrm{P}$ & 1999 \\
\hline Uppsala & Sweden & $\mathrm{P}$ & 1989 \\
\hline Clatterbridge & England & $\mathrm{P}$ & 1989 \\
\hline Loma Linda & CA, USA & $\mathrm{P}$ & 1990 \\
\hline Nice & France & $\mathrm{P}$ & 1991 \\
\hline Orsay & France & $\mathrm{P}$ & 1991 \\
\hline iThemba Labs & South Africa & $\mathrm{P}$ & 1993 \\
\hline IU Health PTC, Bloomington & IN, USA & $\mathrm{P}$ & 2004 \\
\hline UCSF & CA, USA & $\mathrm{P}$ & 1994 \\
\hline HIMAC, Chiba & Japan & C-ion & 1994 \\
\hline TRIUMF, Vancouver & Canada & $\mathrm{P}$ & 1995 \\
\hline HZB (HMI), Berlin & Germany & $\mathrm{P}$ & 1998 \\
\hline NCC, Kashiwa & Japan & $\mathrm{P}$ & 1998 \\
\hline HIBMC, Hyogo & Japan & $\mathrm{P}$ & 2001 \\
\hline HIBMC, Hyogo & Japan & C-ion & 2002 \\
\hline PMRC (2), Tsukuba & Japan & $\mathrm{P}$ & 2001 \\
\hline NPTC, MGH Boston & MA, USA & $\mathrm{P}$ & 2001 \\
\hline INFN-LNS, Catania & Italy & $\mathrm{P}$ & 2002 \\
\hline Shizuoka Cancer Center & Japan & $\mathrm{P}$ & 2003 \\
\hline Southern Tohoku PTC, Fukushima & Japan & $\mathrm{P}$ & 2008 \\
\hline WPTC, Zibo & China & $\mathrm{P}$ & 2004 \\
\hline MD Anderson Cancer Center, Houston & TX, USA & $\mathrm{P}$ & 2006 \\
\hline UFPTI, Jacksonville & FL, USA & $\mathrm{P}$ & 2006 \\
\hline NCC, IIsan & South Korea & $\mathrm{P}$ & 2007 \\
\hline RPTC, Munich & Germany & $\mathrm{P}$ & 2009 \\
\hline ProCure PTC, Oklahoma City & OK, USA & $\mathrm{P}$ & 2009 \\
\hline HIT, Heidelberg & Germany & $\mathrm{P}$ & 2009 \\
\hline HIT, Heidelberg & Germany & C-ion & 2009 \\
\hline UPenn, Philadelphia & PA, USA & $\mathrm{P}$ & 2010 \\
\hline GHMC, Gunma & Japan & C-ion & 2010 \\
\hline IMPCAS, Lanzhou & China & C-ion & 2006 \\
\hline CDH Proton Center, Warrenville & IL, USA & $\mathrm{P}$ & 2010 \\
\hline HUPTI, Hampton & VA, USA & $\mathrm{P}$ & 2010 \\
\hline IFJ PAN, Krakow & Poland & $\mathrm{P}$ & 2011 \\
\hline Medipolis Medical Research Institute, Ibusuki & Japan & $\mathrm{P}$ & 2011 \\
\hline
\end{tabular}

P: proton, C-ion: carbon ion.

are approximately 3 for carbon ion RT, but depend on many factors. Because carbon ion beams are high-LET ion beams, they can create clusters of DNA damage that cannot be repaired, they are effective for the treatment of hypoxic cells, they cause fewer variations in radiosensitivity related to the cell cycle, and fractionated irradiation is less likely to lead to the repair of radiation-induced injury.

2.3. Technical Aspects. Rotating gantries for proton therapy have been installed in more than half of all proton therapy facilities, especially those facilities that have newly opened in the 21st century. Although carbon ion beams have approximately three times the magnetic rigidity of proton beams and are difficult to deflect, they are still delivered in a fixed manner using horizontal or vertical beam lines. The particle therapy facility at the Heidelberger Ionenstrahl-Therapie Centre, Heidelberg, Germany (HIT) has investigated the use of a rotating gantry for carbon ion beams, but this setup has not yet been realized.

Beam delivery has been performed using passive methods with modulators, collimators, and compensators at most facilities. The beam is bended using electromagnets and spread by scattering. A ridge filter alters the width of the Bragg peak, and a range shifter adjusts the beam range. The collimator and bolus regulate the outline and depth of the beam based on the target (the systems vary somewhat among institutions). The advantage of a passive beam delivery system is that the treatment planning for this system is simple and can easily produce a conformal irradiation dose, both 


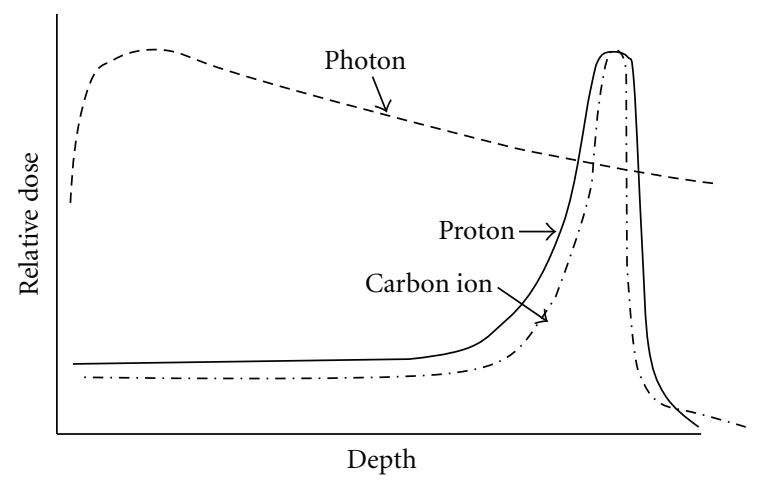

FIGURE 1: Relationship of depth and relative dose in photon, proton and carbon ion beams. Proton and carbon ion beams can produce Bragg peak. The particle range is determined by the energy and larger radiation dose can be delivered to the tumor than photon beam.

laterally and at the correct depth, relative to the target. On the other hand, the main disadvantage is that the area along the entrance path is also unavoidably irradiated, and this area often includes nontarget normal tissue. As an alternative to passive beam delivery, active beam delivery techniques, such as spot scanning or raster scanning, have been developed using narrow pencil beams $[2,3]$. Pencil beams produced in a synchrotron are deflected laterally by two magnetic dipoles, whereas the energy of the incoming beam is varied during the treatment. Thus, a three-dimensional, singlespot Bragg peak is created that conforms to the target after magnetic deflection, and the dose distribution can be tailored optimally to any irregular tumor shape. The dose distribution can also be conformed to the proximal edge of the target volume, and normal tissue that resides along the entrance channel of the beam can thus be spared (Figure 2).

2.4. Financial Aspects. Although particle beam therapy has great physical and biological advantages, only a few facilities throughout the world can perform this treatment, as mentioned above. One of the most important disadvantages of particle beam therapy is the high cost of its technical realization and operation. Large cyclotrons or synchrotrons are needed to accelerate protons and carbon ions to the required energy levels for the treatment of deep-seated tumors. A cost analysis study in Europe calculated the capital costs of proton beam RT to be approximately $€ 60-80$ million and that of carbon ion beam RT to be $€ 160$ million [4]. The operation costs are €16-18 million per year for both proton and carbon ion beam RT. Furthermore, extremely precise and reproducible patient positioning coupled with high-quality imaging for treatment planning is prerequisites for this type of treatment.

\section{Skull Base Tumors}

Chordoma and chondrosarcoma are the most common skull base tumors to be treated using particle beam therapy [5-16] (Table 2). Chordoma and chondrosarcoma are characterized by a high histologic differentiation, locally aggressive growth,

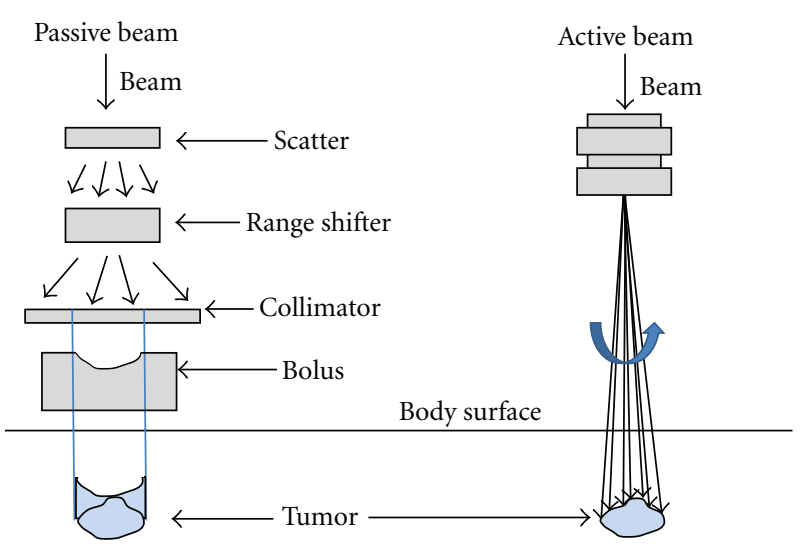

FIGURE 2: Schema of passive (left) and active (right) particle beams (the systems vary somewhat among institutions).

and high recurrence rates. Surgery is the first choice for the treatment of skull base tumors. However, complete resection is difficult and challenging because of the proximity to vulnerable critical organs, such as the optic nerve and brainstem. These anatomic structures often limit surgical resection, and residual tumor tissue results in a high recurrence rate. In addition, many patients are reluctant to undergo gross surgery for cosmetic reasons. Metastasis to lymph nodes in the neck is uncommon for these tumors. Hence, prophylactic neck irradiation is not necessary, and an involved field technique is usually used for RT. Gadolinium contrast magnetic resonance imaging (MRI) offers better soft tissue contrast, which is the most important feature in radiation treatment planning, and diffusion-weight MRI or fluorine-18 deoxyglucose $\left({ }^{18} \mathrm{FDG}\right)$ positron emission tomography imaging can provide important information regarding tumor delineation and activity. The investigation of focal dose escalation to substantially inhomogeneous tumors using ${ }^{18} \mathrm{FDG}$ or other tracers, such as fluorine18 fluoromisonidazole, that are suited for the detection of hypoxic cells has been performed for head and neck cancers; however, useful clinical evidence has not yet been obtained.

Munzenrider reported the long-term follow-up data for 519 patients with chordoma or low-grade chondrosarcoma who were treated with proton beams and photons [6]. The patients received $66-83 \mathrm{GyE}$, and the overall survival rate (OS) at 10 years was $54 \%$ for the patients with chordomas and $88 \%$ for the patients with chondrosarcomas. Myelopathy, neuropathy, hearing loss, and endocrinopathy were severe side effects. Colli reported that chordoma patients treated with proton beams with or without photons had a significantly higher recurrence-free survival rate than patients treated with photons in 2001 [7]. Many clinical studies have been performed using proton and carbon ion beams for the treatment of skull base tumors during this past decade. HIT has traditionally reported updated data for skull base tumors $[9,11,14,17]$. Schulz-Ertner reported that the three-year OS was $91 \%$ and the local control rate (LC) was $81 \%$ in chordoma patients and that the three-year OS was $98 \%$ and the LC was $96 \%$ in chondrosarcoma patients; severe late side effects were observed in less than $2 \%$ of the patients 
TABle 2: Particle beam therapy: skull base tumor.

\begin{tabular}{|c|c|c|c|c|c|c|c|}
\hline Author, year & Institution & $N$ & Disease & Conc. TX & Beam & Dose (GyE) & OS \\
\hline $\begin{array}{l}\text { Munzenrider and } \\
\text { Liebsch, } 1999 \text { [6] }\end{array}$ & MGH & 519 & $\begin{array}{l}\text { Chord }(56 \%) \\
\text { Chondro }(44 \%) \\
\end{array}$ & Unknown & $\mathrm{P}+\mathrm{ph}$ & $66-83$ & $\begin{array}{l}\text { Chord: } 54 \%(10 \mathrm{Y}) \\
\text { Chondro: } 88 \%(10 \mathrm{Y})\end{array}$ \\
\hline Nöel et al., 2003 [8] & $\mathrm{CPO}$ & 67 & $\begin{array}{l}\text { Chord }(70 \%) \\
\text { Chondro }(30 \%)\end{array}$ & $\operatorname{Res}(91 \%)$ & $\mathrm{P}+\mathrm{ph}$ & $60-70(67)$ & $\begin{array}{l}\text { Chord: } 88 \%(4 \mathrm{Y}) \\
\text { Chondro: } 75 \%(4 \mathrm{Y})\end{array}$ \\
\hline $\begin{array}{l}\text { Schulz-Ertner et al., } \\
2004 \text { [9] }\end{array}$ & GSI & 54 & Chord & Res $(100 \%)$ & C-ion & $\begin{array}{l}\text { prim: } 60 \text { or } 70 \\
(60) \\
\text { rec: } 45-60(51)\end{array}$ & $91 \%(3 \mathrm{Y})$ \\
\hline Igaki et al., 2004 [10] & Tsukuba & 13 & Chord & Res $(53 \%)$ & $\mathrm{P}+(\mathrm{ph})$ & $69-105(77)$ & $67 \%(5 \mathrm{Y})$ \\
\hline $\begin{array}{l}\text { Schulz-Ertner et al., } \\
2005 \text { [11] }\end{array}$ & GSI & 29 & ACC & Res $(69 \%)$ & $\mathrm{C}+\mathrm{ph}$ & 72 & $76 \%(4 \mathrm{Y})$ \\
\hline $\begin{array}{l}\text { Pommier et al., } 2006 \\
\text { [12] }\end{array}$ & MGH & 23 & ACC & Unknown & $\mathrm{P}+\mathrm{ph}$ & $70-79.1(76.4)$ & $77 \%(5 \mathrm{Y})$ \\
\hline Tsujii et al., 2007 [13] & NIRS & 25 & Chord & Res $(100 \%)$ & C-ion & $48-60.8$ & $86 \%(5 \mathrm{Y})$ \\
\hline $\begin{array}{l}\text { Schulz-Ertner et al., } \\
2007 \text { [14] }\end{array}$ & GSI & 54 & Chondro & $\operatorname{Res}(100 \%)$ & C-ion & $57-60(60)$ & $98.2 \%(4 \mathrm{Y})$ \\
\hline \multirow[t]{2}{*}{ Ares et al., 2009 [15] } & \multirow[t]{2}{*}{ PSI } & \multirow[t]{2}{*}{64} & Chord (66\%) & \multirow[t]{2}{*}{ Unknown* } & \multirow[t]{2}{*}{$\mathrm{P}$} & $\begin{array}{l}\text { Chord: } 67-74 \\
(73.5)\end{array}$ & Chord: 62\% (5 Y) \\
\hline & & & Chondro (34\%) & & & $\begin{array}{l}\text { Chondro: } 63-74 \\
(68.4)\end{array}$ & Chondro: 91\% (5 Y) \\
\hline $\begin{array}{l}\text { Combs et al., } 2009 \\
\text { [16] }\end{array}$ & HIT & 18 & $\begin{array}{l}\text { Chondro }(59 \%) \\
\text { Chord }(41 \%)\end{array}$ & $\operatorname{Res}(52 \%)$ & C-ion & $60-66.6(60)$ & $100 \%$ \\
\hline
\end{tabular}

Abbreviation; N: number, OS: overall survival rate, GyE: Gray equivalent.

Chord: Chordoma, Chondro: Chondrosarcoma, ACC: Adenoid cystic carcinoma, Res: gross or partial resection, P: proton, ph: photon, C-ion: carbon ion, prim: primary, rec: recurrence.

$*$ : Res (100\%) including biopsy.

in their study of more than 50 patients using carbon ion beams $[9,14]$. Combs reported data for young adult patients with chordoma or chondrosarcoma who were treated with carbon ion beams [16]. A total of 18 patients (5-21 years old) were treated with carbon ion beams of 60-66.6 GyE; the OS was $100 \%$, and the local control rate was $94 \%$ in the $3-$ 112-month follow-up period. Severe late side effects were not found. A report of further long-term follow-up data is expected. In total, many patients received a gross or partial resection before radiotherapy, and the irradiation dose was approximately $60-70 \mathrm{GyE}$ and the OS was $80-90 \%$ at $3-5$ years after treatment. Age, tumor volume, extension, surgical history, and visual function at presentation were pointed out as prognostic factors in some reports. Late side effects were rare in all the studies. Pommier et al. reported that 10 out of 23 adenoid cystic carcinoma (ACC) patients who received $70-79.1 \mathrm{GyE}$ of proton beams and photons exhibited a grade 3 neurologic deficiency (seizures in 7 patients and short-term memory loss in 3 patients); however, all the seizures were controlled with medication [12].

In summary, the effectiveness of conventional photon RT is limited in patients with chordomas and chondrosarcomas, and postoperative high-dose proton or carbon ion beam RT exhibits excellent tumor control without late side effects and should be selected as an adequate treatment of choice.

In recent years, the Paul Scherrer Institute (PSI) and HIT have started active proton and carbon ion beam radiotherapy for the treatment of chordoma and chondrosarcoma patients. Active beam particle beam therapy is, theoretically, extremely adequate for the treatment of skull base tumors in which the tumor and critical organs, such as the optic nerve and brainstem, are tangled together. HIT has also started a prospective comparative phase study of proton and carbon ion beams for the treatment of chordoma and low- and intermediate-grade chondrosarcoma $[18,19]$. The results of these studies will be published in the near future.

\section{Nasal Cavity and Paranasal Sinus Cancers}

Squamous cell carcinoma (SCC), malignant melanoma $(\mathrm{MM})$, and ACC are the most common nasal cavity and paranasal sinus cancers to be treated using particle beam therapy [13, 20-25] (Table 3). Surgery or radiotherapy is the treatment of first choice for nasal cavity and paranasal sinus cancers, and combined treatment is recommended for locally advanced cancers. Complete resection is difficult and challenging for the same reason as for skull base tumors, and the use of high-dose curative radiotherapy with or without chemotherapy is increasing. These anatomic structures often limit surgical resection, and residual tumor tissue results in a high recurrence rate. The necessity of prophylactic neck irradiation is controversial because of the variations in tumor histology, site, and volume. However, an involved field technique is frequently used for locally advanced N0 cancers. Gadolinium contrast MRI, diffusion-weight MRI, and ${ }^{18} \mathrm{FDG}$ are useful for treatment planning.

The National Institute of Radiological Sciences, Chiba, Japan (NIRS) reported a dose escalation study examining carbon ion beam RT for head and neck cancer patients [20]. Because this study included many kinds of head and 
TABLE 3: Particle beam therapy: nasal cavity and paranasal sinus cancer.

\begin{tabular}{|c|c|c|c|c|c|c|c|}
\hline Author, year & Institution & $N$ & Disease & Conc. TX & Beam & Dose (GyE) & OS \\
\hline \multirow{2}{*}{$\begin{array}{l}\text { Weber et al., } 2006 \\
{[21]}\end{array}$} & \multirow{2}{*}{$\mathrm{MGH}$} & \multirow{2}{*}{36} & SCC $(28 \%)$ & \multirow{2}{*}{$\operatorname{Res}(78 \%)$} & \multirow{2}{*}{$\mathrm{P}+\mathrm{ph}$} & \multirow{2}{*}{$60.8-77(69.6)$} & \multirow{2}{*}{$90 \%(3 \mathrm{Y})$} \\
\hline & & & $\operatorname{ACC}(28 \%)$ & & & & \\
\hline \multirow{2}{*}{ Tsujii et al., 2007 [13] } & \multirow{2}{*}{ NIRS } & \multirow{2}{*}{$224^{*}$} & MM (36\%) & & \multirow{2}{*}{ C-ion } & \multirow{2}{*}{57.6} & \multirow{2}{*}{$57 \%(3 \mathrm{Y})$} \\
\hline & & & ACC $(29 \%)$ & & & & \\
\hline \multirow{2}{*}{$\begin{array}{l}\text { Tamtruong et al., } \\
2009 \text { [22] }\end{array}$} & \multirow{2}{*}{ MGH } & \multirow{2}{*}{20} & SCC $(50 \%)$ & $\operatorname{Res}(35 \%)$ & \multirow{2}{*}{$\mathrm{P}+\mathrm{ph}$} & \multirow{2}{*}{$66-78(76)$} & \multirow{2}{*}{$53 \%(2 \mathrm{Y})$} \\
\hline & & & $\operatorname{ACC}(35 \%)$ & $\begin{array}{l}\text { Chemo } \\
(30 \%)\end{array}$ & & & \\
\hline \multirow[t]{2}{*}{ Zenda et al., 2010 [23] } & \multirow[t]{2}{*}{ NCC } & \multirow[t]{2}{*}{39} & $\operatorname{SCC}(28 \%)$ & $\begin{array}{l}\text { Chemo } \\
(3 \%)\end{array}$ & \multirow[t]{2}{*}{$\mathrm{P}$} & \multirow[t]{2}{*}{$60-70(65)$} & \multirow[t]{2}{*}{$59 \%(3 \mathrm{Y})$} \\
\hline & & & ONB $(23 \%)$ & & & & \\
\hline Jingu et al., 2011 [24] & NIRS & 37 & MM (100\%) & $\begin{array}{l}\text { Chemo } \\
(100 \%)\end{array}$ & C-ion & 57.6 & $81 \%(3 \mathrm{Y})$ \\
\hline \multirow{2}{*}{$\begin{array}{l}\text { Fukumitsu et al., } 2011 \\
{[25]}\end{array}$} & \multirow[t]{2}{*}{ Tsukuba } & \multirow[t]{2}{*}{17} & SCC (65\%) & Chemo & \multirow{2}{*}{$\mathrm{P}+(\mathrm{ph})$} & \multirow[t]{2}{*}{$72.4-89.6(78)$} & \multirow[t]{2}{*}{$47 \%(2 \mathrm{Y})$} \\
\hline & & & $\mathrm{AC}(12 \%)$ & $(12 \%)$ & & & \\
\hline
\end{tabular}

N: number, OS: overall survival rate, Conc. TX: concurrent treatment, GyE: Gray equivalent.

SCC: squamous cell carcinoma, ACC: adenoid cystic carcinoma, MM: malignant melanoma, ONB: olfactory neuroblastoma, AC: adenocarcinoma, Res: gross or partial resection, Chemo: chemotherapy, P: proton, ph: photon, C-ion: carbon ion. ${ }^{*}$ Including skull base tumor.

neck cancer patients, the outcome of only nasal cavity and paranasal sinus cancer patients is difficult to extract. However, this study revealed that carbon ion RT consisting of $70.2 \mathrm{GyE}$ administered in 18 fractions over 6 weeks and carbon ion RT consisting of $64 \mathrm{GyE}$ administered in 16 fractions over 4 weeks had equal clinical outcomes in terms of morbidity and local control. In addition, the treatment effectiveness differed largely according to tumor histology, and MM had a better LC than SCC.

Previous studies of nasal cavity and paranasal sinus cancer can be divided into two types according to tumor histology: mainly SCC and non-SCC. In a study examining mainly SCC, the irradiation dose was approximately 60 $80 \mathrm{GyE}$ and the OS was $50-90 \%$ at 2-3 years after treatment. The OS was $90 \%$ and the disease-free survival rate was $77 \%$ at 3 years in a study in which $78 \%$ of the patients, who had mainly SCC, received a gross resection before undergoing RT using proton beams and photons $(60.8-77 \mathrm{GyE})$ [21], whereas the OS was $59 \%$ at 3 years and $47 \%$ at 2 years in studies in which no patients received any kind of resection before undergoing RT $[23,25]$. In studies examining nonSCC, the irradiation dose was approximately $50-80 \mathrm{GyE}$ and the OS was $50-80 \%$ at 3 years after treatment. Jingu at the NIRS reported an OS of $81 \%$ and an LC of $65 \%$ among MM patients who were treated with $57.6 \mathrm{GyE}$ of carbon ion beams with concurrent chemotherapy. The tumor volume, site, and extension were identified as prognostic factors in some reports. Overall, reported severe late side effects have included brain necrosis, bone necrosis, and deteriorations in visual function; however, either the morbidity rate was quite low or the morbidities were inevitable and the patients' consensus had been obtained in each of the studies.

In summary, the OS of patients with nasal cavity or paranasal sinus cancer varies widely. The rarity of such tumors makes it difficult to perform large studies and may cause a patient bias at each institute. Consequently, comparisons among studies are also difficult. Further studies involving larger numbers of patients are needed.

\section{Discussion and Future Directions}

Particle therapy with protons and carbon ion beams offers physical and biological advantages, compared with conventional photon RT. On the other hand, a complex technique is needed, and the capital and operation costs are relatively high. Particle beam therapy is advantageous for the treatment of tumors such as skull base, nasal cavity, and paranasal sinus cancers, as these tumors are often located close to critical organs. A clinical review has revealed that highdose irradiation is a feasible and safe treatment. To further improve the applicability of particle beam therapy, future developments in the fields of imaging and radiation technology must be fully integrated into the particle therapy process. For example, most modern treatment planning systems include advanced image registration functions. As imaging technologies improve, intensive irradiation dose planning will become more accurate, and an active beam delivery technique will be able to deliver a focally intensive radiation dose to the tumor while avoiding critical organs in the vicinity. Regarding radiation technology, a rotating gantry of carbon ion beams, which is presently under investigation, will be able to overcome the disadvantage of carbon ion beams and should be capable of excellent treatment effects once it is realized.

Some clinical studies have examined the treatment of skull base, nasal cavity, and paranasal sinus cancer patients using particle beam therapy. However, because of the rarity of such tumors, definite conclusions were not obtained. Prospective trials involving a large number of patients are needed to obtain clinical evidence. Moreover, past studies have mainly focused on OS, LC, and side effects. In recent 
years, photon RT is also technically progressing especially development of intensive modulated radiotherapy (IMRT). To apply particle beam therapy widely, a cost performance analysis comparing conventional RT or IM RT is very important in the future studies.

\section{References}

[1] R. R. Wilson, "Radiological use of fast protons," Radiology, vol. 47, no. 5, pp. 487-491, 1946.

[2] H. Eickhoff, T. Haberer, G. Kraft et al., "The GSI cancer therapy project," Strahlentherapie und Onkologie, vol. 175, no. 2, pp. 21-24, 1999.

[3] E. Pedroni, R. Bacher, H. Blattmann et al., "The 200-MeV proton therapy project at the Paul Scherrer Institute: conceptual design and practical realization," Medical Physics, vol. 22, no. 1, pp. 37-53, 1995.

[4] A. Peeters, J. P. C. Grutters, M. Pijls-Johannesma et al., "How costly is particle therapy? Cost analysis of external beam radiotherapy with carbon-ions, protons and photons," Radiotherapy and Oncology, vol. 95, no. 1, pp. 45-53, 2010.

[5] J. Debus, E. B. Hug, N. J. Liebsch et al., "Brainstem tolerance to conformal radiotherapy of skull base tumors," International Journal of Radiation Oncology Biology Physics, vol. 39, no. 5, pp. 967-975, 1997.

[6] J. E. Munzenrider and N. J. Liebsch, "Proton therapy for tumors of the skull base," Strahlentherapie und Onkologie, vol. 175, no. 2, pp. 57-63, 1999.

[7] B. O. Colli and O. Al-Mefty, "Chordomas of the craniocervical junction: follow-up review and prognostic factors," Journal of Neurosurgery, vol. 95, no. 6, pp. 933-943, 2001.

[8] G. Noël, J. L. Habrand, E. Jauffret et al., "Radiation therapy for chordoma and chondrosarcoma of the skull base and the cervical spine: prognostic factors and patterns of failure," Strahlentherapie und Onkologie, vol. 179, no. 4, pp. 241-248, 2003.

[9] D. Schulz-Ertner, A. Nikoghosyan, C. Thilmann et al., "Results of carbon ion radiotherapy in 152 patients," International Journal of Radiation Oncology Biology Physics, vol. 58, no. 2, pp. 631-640, 2004.

[10] H. Igaki, K. Tokuuye, T. Okumura et al., "Clinical results of proton beam therapy for skull base chordoma," International Journal of Radiation Oncology Biology Physics, vol. 60, no. 4, pp. 1120-1126, 2004.

[11] D. Schulz-Ertner, A. Nikoghosyan, B. Didinger et al., "Therapy strategies for locally advanced adenoid cystic carcinomas using modern radiation therapy techniques," Cancer, vol. 104, no. 2, pp. 338-344, 2005.

[12] P. Pommier, N. J. Liebsch, D. G. Deschler et al., "Proton beam radiation therapy for skull base adenoid cystic carcinoma," Archives of Otolaryngology, vol. 132, no. 11, pp. 1242-1249, 2006.

[13] H. Tsujii, J. Mizoe, T. Kamada et al., "Clinical results of carbon ion radiotherapy at NIRS," Journal of Radiation Research, vol. 48, pp. A1-A13, 2007.

[14] D. Schulz-Ertner, A. Nikoghosyan, H. Hof et al., "Carbon ion radiotherapy of skull base chondrosarcomas," International Journal of Radiation Oncology Biology Physics, vol. 67, no. 1, pp. 171-177, 2007.

[15] C. Ares, E. B. Hug, A. J. Lomax et al., "Effectiveness and safety of spot scanning proton radiation therapy for chordomas and chondrosarcomas of the skull base: first long-term report,"
International Journal of Radiation Oncology Biology Physics, vol. 75, no. 4, pp. 1111-1118, 2009.

[16] S. E. Combs, A. Nikoghosyan, O. Jaekel et al., "Carbon ion radiotherapy for pediatric patients and young adults treated for tumors of the skull base," Cancer, vol. 115, no. 6, pp. 13481355, 2009.

[17] D. Schulz-Ertner, A. Nikoghosyan, C. Thilmann et al., "Carbon ion radiotherapy for chordomas and low-grade chondrosarcomas of the skull base. Results in 67 patients," Strahlentherapie und Onkologie, vol. 179, no. 9, pp. 598-605, 2003.

[18] A. V. Nikoghosyan, I. Karapanagiotou-Schenkel, M. W. Münter, A. D. Jensen, S. E. Combs, and J. Debus, "Randomised trial of proton vs. carbon ion radiation therapy in patients with chordoma of the skull base, clinical phase III study HIT1-Study," BMC Cancer, vol. 10, article 607, 2010.

[19] A. V. Nikoghosyan, G. Rauch, M. W. Münter et al., "Randomised trial of proton vs. carbon ion radiation therapy in patients with low and intermediate grade chondrosarcoma of the skull base, clinical phase III study," BMC Cancer, vol. 10, article 606, 2010.

[20] J. E. Mizoe, H. Tsujii, T. Kamada et al., "Dose escalation study of carbon ion radiotherapy for locally advanced head-andneck cancer," International Journal of Radiation Oncology Biology Physics, vol. 60, no. 2, pp. 358-364, 2004.

[21] D. C. Weber, A. W. Chan, S. Lessell et al., "Visual outcome of accelerated fractionated radiation for advanced sinonasal malignancies employing photons/protons," Radiotherapy and Oncology, vol. 81, no. 3, pp. 243-249, 2006.

[22] M. Tamtruong, U. R. Kamat, N. J. Liebsch et al., "Proton radiation therapy for primary sphenoid sinus malignancies: treatment outcome and prognostic factors," Head and Neck, vol. 31, no. 10, pp. 1297-1308, 2009.

[23] S. Zenda, R. Kohno, M. Kawashima et al., "Proton Beam Therapy for Unresectable Malignancies of the Nasal Cavity and Paranasal Sinuses," International Journal of Radiation Oncology, Biology, Physics, vol. 81, no. 5, pp. 1473-1478, 2010.

[24] K. Jingu, R. Kishimoto, J. E. Mizoe et al., "Malignant mucosal melanoma treated with carbon ion radiotherapy with concurrent chemotherapy: prognostic value of pretreatment apparent diffusion coefficient (ADC)," Radiotherapy and Oncology, vol. 98, no. 1, pp. 68-73, 2011.

[25] N. Fukumitsu, T. Okumura, M. Mizumoto et al., "Outcome of T4 (UICC 7th) or recurrent nasal cavity and paranasal sinus carcinoma treated with proton beam," International Journal of Radiation Oncology, Biology, Physics, vol. 83, no. 2, pp. 704$711,2012$. 


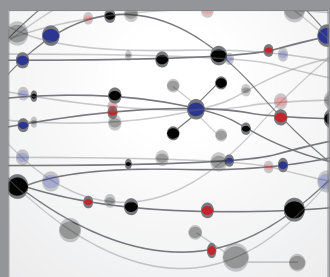

The Scientific World Journal
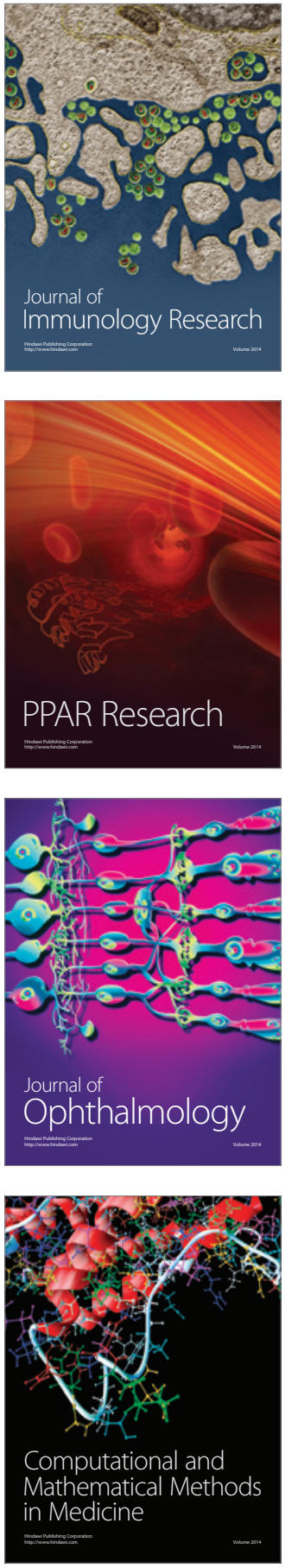

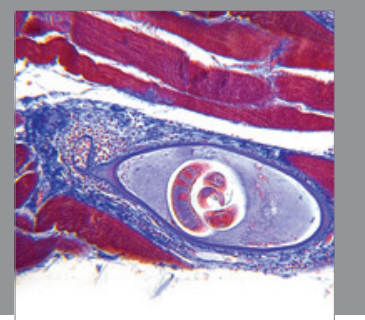

Gastroenterology

Research and Practice
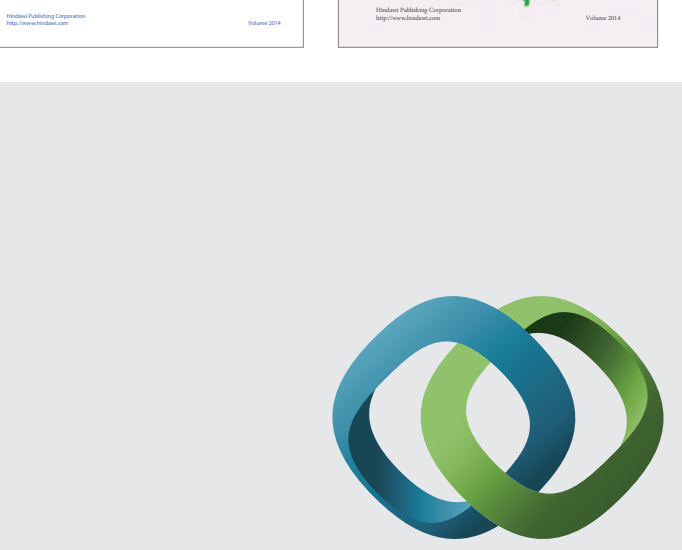

\section{Hindawi}

Submit your manuscripts at

http://www.hindawi.com
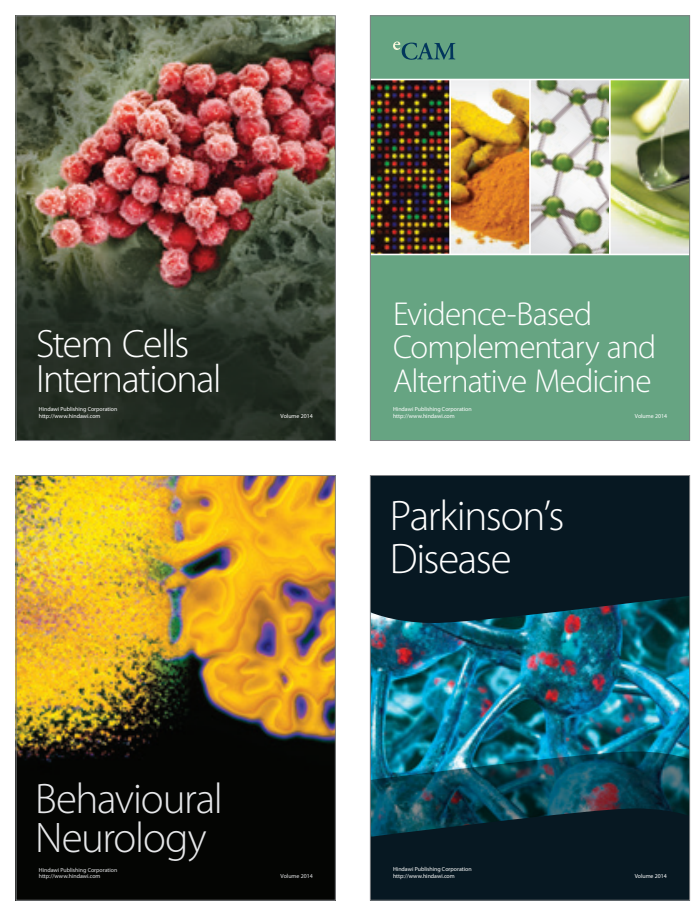

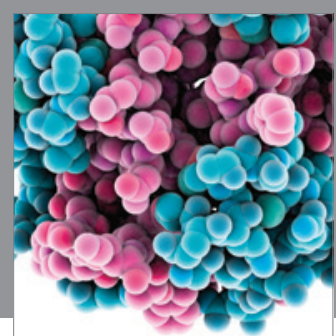

Journal of
Diabetes Research

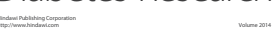

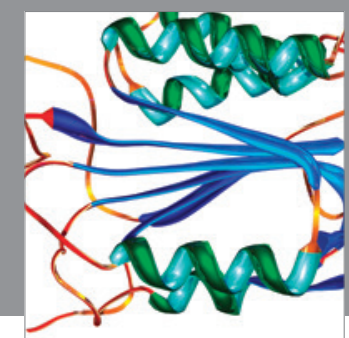

Disease Markers
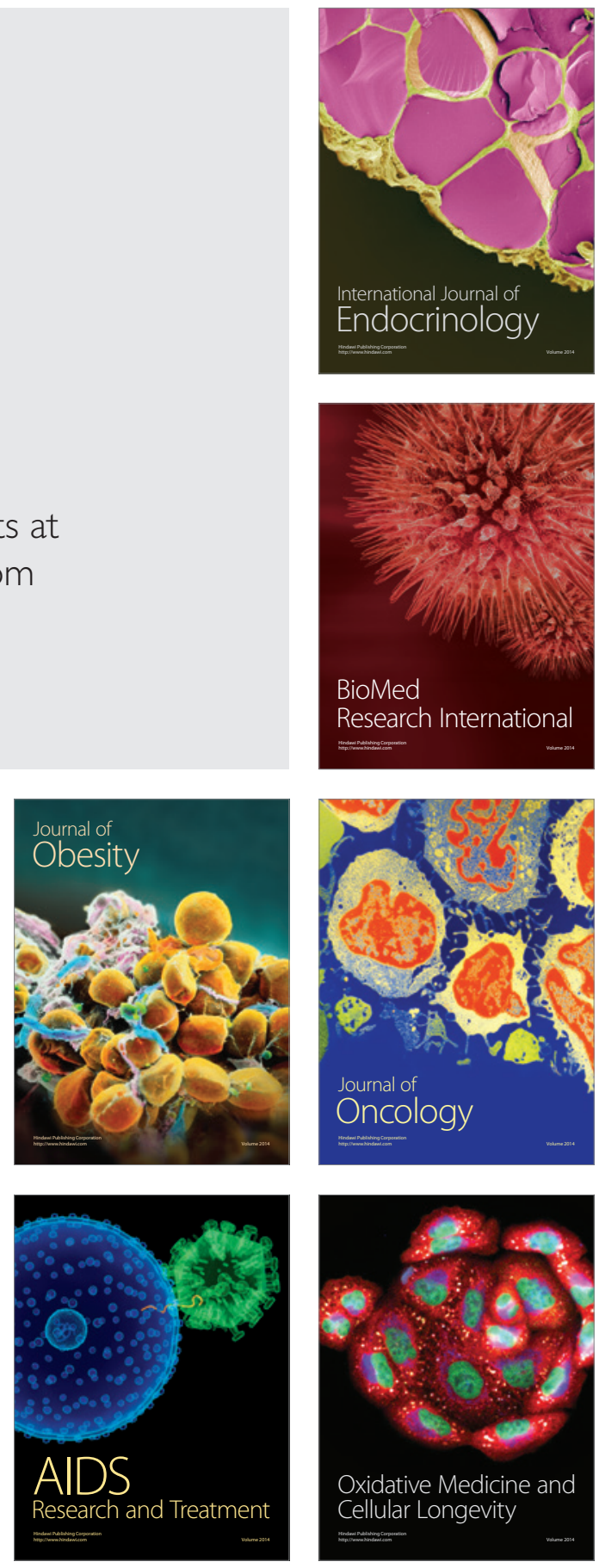